Tropical Journal of Pharmaceutical Research October 2020; 19 (10): 2179-2185

ISSN: $1596-5996$ (print); 1596-9827 (electronic) (C) Pharmacotherapy Group, Faculty of Pharmacy, University of Benin, Benin City, 300001 Nigeria.

\title{
Role of modafinil in the treatment of patients with methamphetamine dependence; An update on randomized, controlled clinical trial
}

\author{
Mahnoosh Tavakkoli Fard ${ }^{1}$, Saeid Sadat Mansouri ${ }^{2 *}$, Alireza Jafari ${ }^{2}$, Nasim \\ Vousooghi ${ }^{1,3}$ \\ ${ }^{1}$ Tehran University of Medical Science, Tehran, Inflammatory Lung Diseases Research Center, Department of Internal \\ Medicine, '2Urology Research Center, Razi Hospital, School of Medicine, Guilan University of Medical Sciences, Rasht, \\ ${ }^{3}$ Research Center for Cognitive and Behavioral Sciences, Tehran University of Medical Science, Tehran, Iran
}

*For correspondence: Email: sahand8141557@yahoo.com

Sent for review: 6 September 2019

Revised accepted: 19 September 2020

\begin{abstract}
Purpose: Methamphetamine is a powerful, highly addictive stimulant which affects the central nervous system. Here we evaluated the efficacy of modafinil for the treatment of patients with MA dependence. Methods: A randomized parallel controlled trial study was designed to compare the effectiveness of take-home, self-administered modafinil treatment in the intervention and the controlled group in three months. The primary outcome measurements were severity and duration of craving, and self-reported methamphetamine consumption, which was confirmed by urine drug test.

Results: The relapse rate in the intervention group was $40 \%$, and the controlled group was $75 \%$, indicating a decrease in relapse rate of MA in the intervention group. During the three months, the severity and the duration of the drug abuse craving in the intervention group were less than the controlled group. Patients in the intervention group experienced an increase in the retention rate and a decrease in the slip rate.

Conclusion: The use of modafinil is not only effective on craving and relapse reduction, but also changes urine drug screens of patients with MA dependence to negative. The modafinil is safely indicated as an absolutely effective medication to reduce withdrawal symptoms and the craving of patients with MA dependence.
\end{abstract}

Keywords: Modafinil, Methamphetamine dependence, Effectiveness, Clinical trial

\begin{abstract}
This is an Open Access article that uses a fund-ing model which does not charge readers or their institutions for access and distributed under the terms of the Creative Commons Attribution License (http://creativecommons.org/licenses/by/4.0) and the Budapest Open Access Initiative (http://www.budapestopenaccessinitiative.org/read), which permit unrestricted use, distribution, and reproduction in any medium, provided the original work is properly credited.

Tropical Journal of Pharmaceutical Research is indexed by Science Citation Index (SciSearch), Scopus, International Pharmaceutical Abstract, Chemical Abstracts, Embase, Index Copernicus, EBSCO, African Index Medicus, JournalSeek, Journal Citation Reports/Science Edition, Directory of Open Access Journals (DOAJ), African Journal Online, Bioline International, Open-J-Gate and Pharmacy Abstracts
\end{abstract}

\section{INTRODUCTION}

Addiction is a major problem around the world that directly or indirectly affects a large percentage of the population. The prevalence of amphetamine type stimulant (ATS) intake in the world is estimated at $0.7 \%$. Unfortunately, in recent years, the prevalence of ATS in Asian countries has grown significantly. In Iran, before 2007, methamphetamine consumption was very limited, and its peak consumption started in 2008 with its domestic production. Despite advances in the treatment of drug dependence, recurrence or uncontrolled drug use is one of the major issues 
in substance dependence. The most effective treatment for methamphetamine dependency is psychological treatment of the matrix, an Iranian version of which was presented by the Iranian National Center of Addiction Studies in 2009. However, this treatment also fails in many cases and the effective therapeutic treatment in the treatment of these patients is still absent [1]. One of the drugs that can be the perfect choice for the treatment of methamphetamine dependence is an amphetamine-stimulating agent, modafinil whose observed clinical effects can improve withdrawal symptoms. Another group of studies that examines the acute physiological and behavioral effects of modafinil in drug addicts shows that it is not abusive [2].

In the current study, the efficacy of take-home self-administration weekly modafinil (with weekly clinical review), in terms of slipping from treatment, relapse, retention rate and mental health indicators to a control condition for individuals with a methamphetamine dependence was compared [3].

\section{METHODS}

\section{Design of the study}

A randomized parallel controlled trial study (phase II) was designed to compare the effectiveness of take-home self-administered modafinil treatment over a three-month period. The International Ethical Guidelines for Healthrelated Research prepared by the Council for International Organizations of Medical Sciences (ClOMS) in collaboration with the World Health Organization (WHO) was used to this study [4]. Forty participants were included in the trial in the outpatient addiction treatment clinics (Rasht, Iran). During the trial, randomized participants were given access to modafinil or basic treatment including cognitive behavioral therapy (CBT) counseling and other medications except for modafinil. The protocol of study was approved by Tehran University Medical Center Institutional Review Board (no. IR.GUMS.REC.1397.513).

\section{Samples}

The participants were males, aged 18 years, or older. Methamphetamine dependence diagnostic criteria (at least 20 of the 28 days) were used for participants based on diagnostic and statistical manual of mental disorders (DSM5). Participants were required to be treatment seeking (by selfreport), and to be likely to complete the threemonth treatment period. Exclusion criteria included diagnosed dependence on any psychoactive substance other than cocaine, alcohol, benzodiazepines, or marijuana; concurrent major medical or psychiatric conditions where immediate opioid agonist treatment and/or other treatments were clinically indicated. Participants were recruited for treatment from the addiction treatment clinics in Rasht, Guilan, Iran, from April 2014 to March 2015. Participants completed screening with the research nurses. They confirmed participants' eligibility, including urine drug testing, by ACON (12 Panel Drug Screening Test Card) rapid test. It was anticipated that in the event of drug allergy and unwanted effects at each stage, the study would be stopped and the participants would be excluded. However, no complications were observed during the study. The research nurses or research assistants obtained voluntary written consent from all participants and completed study enrollment procedures.

\section{Procedures}

Independent researchers carried out random number allocation, block randomization for the trial with a 1:1 allocation ratio. Participants and researchers were unaware of the randomization. Based on similar studies $(5,6)$, fifty and five methamphetamine dependent patients were volunteered for treatment. After screening, fifteen patients were excluded from the study due to coadministration of other substances, medical conditions, or psychiatric illnesses. Forty eligible patients were randomized to receive modafinil or other medication except for modafinil. Subjects participated in a three-month treatment phase and a three-month follow-up period that concluded with an end-of-study visit in six months. Participants permuted block randomized to treatment were inducted with a dose of 100 $\mathrm{mg}$ of modafinil on the first day, with $200 \mathrm{mg}$ take-home medication provided for the second day. The research physicians reviewed participants on the second day to assess medication adherence, dose adequacy, drug use, and cravings, and they changed the dose, if necessary. Doses were clinically titrated with the goal of a target dose of $300 \mathrm{mg}$ modafinil (maximum dose), and provided doses for days three to seven. The research physicians reviewed all treatment participants each week and assessed progress during a $15-20 \mathrm{~min}$ standardized medical management session. Medical management sessions included participant confirmation of dose amount questions relating to side effects and adverse event reporting if required, rating of any cravings, participant self-report of modafinil and illicit drug use in the past week, recording of concomitant medication and supervised urine collection. Throughout the three months of treatment, 
modafinil group participants attended the clinic weekly and one weeks' take-home medication was provided for self-administration.

Dose titration could occur during each medical review session (at the end of weeks) and was based on clinical assessment that comprised patient self-reported other drug use, cravings, physical examination, urine drug screen results and assessment of patient progress in treatment. Since outpatient and self-administered treatment was performed, a number of patients dropped out of treatment in each stage. Research assessments were conducted for both groups at base line and at the end of first month, third month, and the sixth month. During the follow-up period, medical assessments were performed and methamphetamine urine samples were collected. The screening instruments were administered: The General Health Questionnaire (GHQ, self-reported), severity of withdrawal symptoms, craving severity and temporality checklists, and urine drug screens test. Participants' urine drug screens were collected on a weekly basis.

\section{Outcomes}

The primary outcome was self-reported methamphetamine use confirmed by urine toxicology, severity, and duration of craving measured by craving severity and temporality checklists, severity of withdrawal symptoms measured by ATS withdrawal symptoms checklist. The secondary outcomes for this trial were other substance use such as morphine, alcohol, benzodiazepines, marijuana, physical and mental health measured by GHQ questionnaire. Retention rate was another outcome of this trial. All primary and secondary outcomes were measured at the beginning of the study, the end of the first and the third month. An independent clinical monitoring committee, comprising addiction medicine specialists assessed progress of participants and had the authority to remove participants from either trial condition if their health status deteriorated significantly during the trial.

\section{Statistical analysis}

For the effectiveness analysis, continuous data have been summarized using descriptive statistics including the number of observations used in the calculation, mean, and standard deviation (SD). Categorical data have been summarized as counts and percentages of each category. Analyses were performed on those who were randomized to the modafinil program and another group. For continuous and count outcomes, between-group differences at each time-point were assessed using linear regression, and a random intercept to account for the serial correlation induced from longitudinal measurements; results are presented as means with $95 \%$ confidence intervals.

\section{RESULTS}

The study design is presented in figure 1 . Participants were recruited from April 2014 to March 2015. Fifty-five patients were screened for entry to the study. Fifteen subjects were not eligible - most commonly for not completing the assessment process. Forty patients were eligible and signed the consent form.

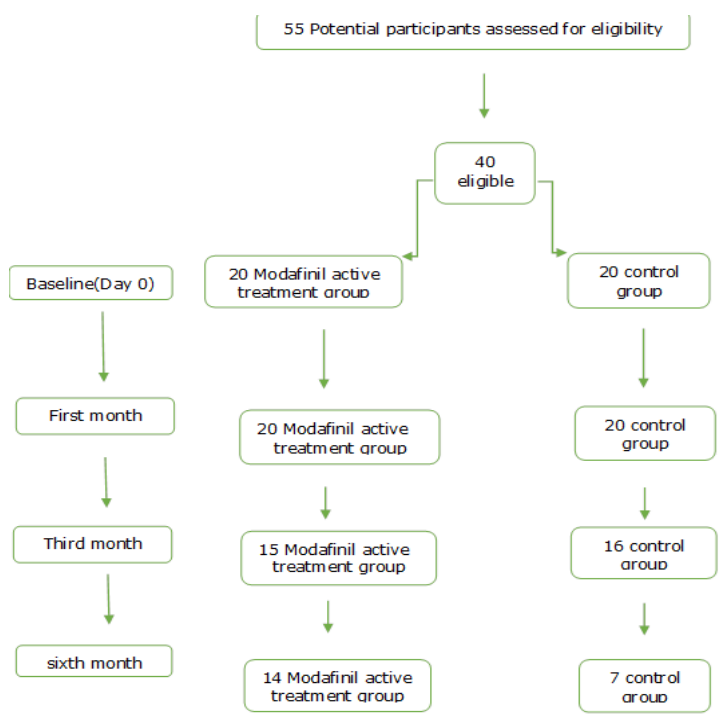

Fig 1: Trial profile, recruitment, and progress of study participants.

Trial subjects were assessed at baseline (day zero), and at the end of the first, Third, and sixth month for MA use, other substance use, general health, craving, and retention rate. Participant numbers at each phase of the protocol are provided. Patients' demographic data are presented in Table 1.

The mental health index of patients at the onset of treatment and follow-up on the first and third months are presented in Table 2.

The mental health index of patients at the beginning of the study in the group prescribed for them was not significantly different from that of the control group. However, one month later and in the follow up of the three months, this index had a significant difference in the two groups; 
Table 1: Demographics characteristics and stratification factors of participant

\begin{tabular}{|c|c|c|c|}
\hline Variable & $\begin{array}{l}\text { Modafinil group } \\
(n=20)\end{array}$ & $\begin{array}{c}\text { Control group } \\
(n=20)\end{array}$ & $\begin{array}{c}\text { Total } \\
(n=40)\end{array}$ \\
\hline Gender, male, n(\%) & $20(100 \%)$ & $20(100 \%)$ & $40(100 \%)$ \\
\hline Age, years, mean (SD) & $32.35(8.04)$ & $29.65(7.07)$ & $31(7.89)$ \\
\hline 12 years $^{+}$education, $n$ (\%) & $13(65 \%)$ & $9(45 \%)$ & $22(55 \%)$ \\
\hline Employed, n (\%) & $17(85 \%)$ & $15(75 \%)$ & $32(80 \%)$ \\
\hline $\begin{array}{l}\text { Marital status } \\
\text { (Married, Single, Divorced) }\end{array}$ & $\begin{array}{c}11(55 \%), 7(35 \%), \\
2(10 \%)\end{array}$ & $\begin{array}{l}5(25 \%), \\
3(15 \%)\end{array}$ & $\begin{array}{c}16(40 \%), 19(47 \%), \\
5(12 \%)\end{array}$ \\
\hline
\end{tabular}

Table 2: Mental health index at the onset of treatment and follow-up stages

\begin{tabular}{llcccc}
\hline Variable & Group & N & Mean & SD & $P$-value \\
\hline GHQ (baseline) & Modafinil group & 20 & 36.65 & 14.14 & 0.576 \\
& Control group & 20 & 38.60 & 11.10 & \\
GHQ (1st month) & Modafinil group & 20 & 24.60 & 12.53 & $0.019^{*}$ \\
& Control group & 20 & 33.80 & 11.29 & \\
GHQ (3rd month) & Modafinil group & 15 & 12.07 & 5.70 & $0.014^{*}$ \\
& Control group & 16 & 21.38 & 12.55 & \\
\hline
\end{tabular}

the modafinil group had a considerable improvement compared to the control group that could be due to the effect of modafinil on improving these indicators $(P$-value $<0.05)$.

In monitoring the retention rate, $70 \%$ of patients in the modafinil group, remained in treatment at least six months, while in the control group, only $35 \%$ of patients were, and $65 \%$ of them remained in treatment for only less than three months (Fig 2).

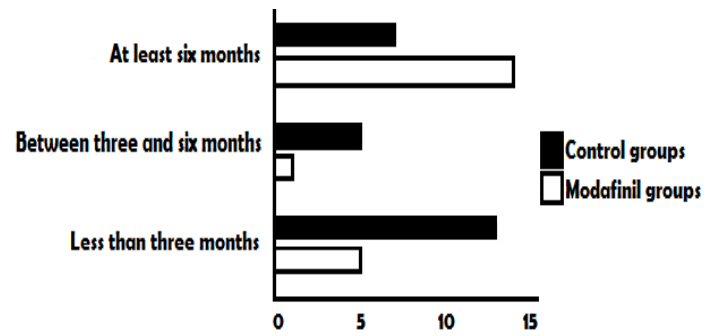

Figure 2: Distribution of participant based on retention rate in treatment in both case and control groups

As shown in Table 3, Table 4 and Table 5, there was no significant difference in the severity of withdrawal symptoms at the beginning of the study in the two groups, modafinil and control group. One month after treatment, there was a significant decrease in the number of symptoms in the modafinil group $(p<0.05)$. Some of these symptoms, which were reported by patients in the previous studies, were the main causes of relapse and slipping from treatment, such as hypersomnia, decreased activity, lethargy, and severity and duration of craving that were significantly improved with the use of modafinil. In addition, the severity of withdrawal symptoms in the follow-up of the third month after treatment in the modafinil group was significantly lower than that of the control group $(p<0.05)$. As is demonstrated in Table 4, the duration of craving, and in Table 5 , the severity of craving at the onset of treatment was approximately the same in the two groups. However, the data showed a steep decline in the modafinil group, so that in the follow up of the first and third months, the two groups were significantly different in terms of severity and duration of craving $(P$-value $<0.05)$.

Based on Table 4 and Table 5 , the severity of withdrawal symptoms, the duration of craving and the severity of craving at the onset of treatment in the two groups were approximately the same. However, the charts of all three indices showed a steep decline in the modafinil group, so that in the follow up of the first and third months, the two groups showed a significant difference in terms of withdrawal symptoms, severity, and duration of craving.

Table 3: Monitoring of severity of withdrawal symptoms in three stages

\begin{tabular}{llllcc}
\hline Variable & Group & N & Mean & SD & P-value \\
\hline Severity of withdrawal symptoms & Modafinil group & 20 & 50.40 & 4.333 & \multirow{2}{*}{0.794} \\
(baseline) & Control group & 20 & 50.75 & 4.064 & \\
Severity of withdrawal symptoms (1st & Modafinil group & 20 & 33.00 & 6.743 & \multirow{2}{*}{0.000} \\
month) & Control group & 20 & 44.75 & 6.942 & \\
Severity of withdrawal symptoms (3rd & Modafinil group & 15 & 24.57 & 2.793 & \multirow{2}{*}{0.000} \\
month) & Control group & 16 & 39.50 & 5.833 & \\
\hline
\end{tabular}


Table 4: Duration of Craving at the onset of treatment and follow-up periods

\begin{tabular}{llcccc}
\hline Variable & Group & N & Mean & SD & P-value \\
\hline Duration of craving (baseline) & Modafinil group & 20 & 5.55 & 1.146 & 0.622 \\
& Control group & 20 & 5.35 & 1.387 & \\
Duration of craving (1st month) & Modafinil group & 20 & 3.10 & .788 & 0.040 \\
& Control group & 20 & 3.75 & 1.118 & \\
Duration of craving (3rd month) & Modafinil group & 15 & 1.67 & .724 & 0.000 \\
& Control group & 16 & 3.00 & 1.095 & \\
\hline
\end{tabular}

Table 5: Craving severity index at the onset of treatment and follow-up periods

\begin{tabular}{llcccc}
\hline Variable & Group & N & mean & SD & $P$-value \\
\hline Craving severity (baseline) & Modafinil group & 20 & 5.75 & 1.118 & 0.134 \\
& Control group & 20 & 5.15 & 1.348 & \\
Craving severity (1st month) & Modafinil group & 20 & 3.05 & .826 & 0.010 \\
& Control group & 20 & 4.10 & 1.483 & \\
Craving severity (3rd month) & Modafinil group & 15 & 1.47 & .516 & 0.000 \\
& Control group & 16 & 3.06 & 1.181 & \\
\hline
\end{tabular}

Compared to controls, significant and sustained improvements were seen in the treatment group at all-time points and as an effect over time for quality of life and mental health. The findings showed that $25 \%$ of the patients in the modafinil group during the three-month treatment period and the three-month follow up, there was no slippage. However, in the control group, all patients had at least one slip. Also, in the modafinil group, the relapse was $40 \%$ after 6 months (vs. $75 \%$ in controls). Therefore, modafinil reduces cases of slipping and relapse in MA dependent patients.

Regarding the urine screening tests, the trial was designed to obtain 12 urines from each patient during medication treatment. Missing urines were imputed as positive. The obtained percentage of the expected urines for the modafinil group was $55.5 \%$, and for the control group was $48.5 \%$; a ttest showed that the average number of urines provided was significantly different across the two groups $(\mathrm{t}=2.61, p<0.05)$.

\section{DISCUSSION}

This study demonstrated that in comparison to routine CBT treatment ran in the control group, dependent participants who were treated with modafinil achieved a significant reduction in methamphetamine use, and improvement of mental health over a quarterly period. As mentioned, the decrease in self-reported methamphetamine use in the modafinil group was supported by a significant reduction in return of positive drug screens for MA compared to controls at third and sixth months. The modafinil group demonstrated an immediate response in reduced MA positive urine screens at week one that continued throughout the study. The treatment effect may have been over-estimated in the modafinil group as sampling was done every week, however, the significant positive impact over time with regards illicit MA, or other ATS use during the treatment period was notable. The findings showed that modafinil was effective in reducing relapse and slipping. These findings are consistent with research findings suggesting that modafinil reduced MA seeking behavior and was recommended as an effective treatment for relapse prevention [7]. In another study, modafinil has been shown to have milder deprivation symptoms in the avoidance of MA [8].

Comparison of severity and duration of craving in the modafinil group and the control group showed that these two variables in the modafinil group was lower than the control group. These findings are consistent with the findings of Kampman [9]. Modafinil is considered effective in reducing the craving for cocaine [9]. In addition, the results of this study are consistent with the results of the study by Anderson et al and some of other researcher who showing that taking modafinil with psychotherapy has been effective in reducing cocaine craving $[10,11]$. Undoubtedly, results are inconsistent with the study of Heinzerling based on the lack of efficacy of modafinil on craving for methamphetamine in MA dependent patients has been reported, although in this study the small sub-group of modafinil was able to increase retention rate ]12]. Regarding the rate of relapse and slip in the modafinil group and control, the findings showed that modafinil was effective in reducing recurrence and slipping. These findings are consistent with the findings of Reichel, which suggested that modafinil reduced the MA seeking behavior and were recommended as an effective treatment for the prevention of relapse during abstinence [7]. Comparison of psychosocial indices in methamphetaminedependent patients in the two groups of modafinil and the control group showed that although 
mental health indicators did not differ significantly between the two groups at the onset of treatment, as time and progression went by, the subscale was significantly better in the modafinil group than in the control group. These results coincided with the study by González, which indicated that modafinil improves MA cognitive deficits (13). In addition, in the study of Kalechstein, modafinil was able to improve the functional memory in MA-dependent patients who have had memory loss during abrasion (14). On the other hand, in the study of McGregor modafinil also led patients to experience milder deprivation during MA absenteeism, while sleep disorders were also lower in the modafinil group than in the control group (8). The current study also demonstrated that the retention rate in the patients with MA-dependence in the modafinil group was greater than the control group. The results of this study are comparable with the study by Lee, about the efficacy of modafinil on the therapeutic components of 20 MA-dependent patients(15). They concluded that $67 \%$ of the patients in the modafinil group and $50 \%$ of the patients in the control group completed the course of treatment, although this difference was not statistically significant. Of course, the results of Nicole Lee research can be influenced by the small sample size(16). Also, in a study by Heinzinger, it was shown that the retention rate in patients with $400 \mathrm{mg}$ of modafinil, with more used-days in the last 30 days (more than 18 days from 30 days) was more effective than in patients with less used-days(12). In another study, McElhiney and Rabkin showed the efficacy of modafinil in reducing relapse, craving, and increase retention in treatment, respectively $(17,18)$. The results of this study are consistent with the results of Shearer to increase the negative MA urine test in dependent patients treated with modafinil compared to the control group (19). In another study, Karila, found that Modafinil was an effective medication to reduce craving and to increase the percentage of negative urine tests in terms of MA (20).

Considering the different results that have been obtained from various studies in the world, many studies emphasized the efficacy of modafinil in the treatment of methamphetamine dependence; but these studies seem to be insufficient to achieve the final result. Similar studies have to be done with larger sample sizes in different parts of the world to achieve more reliable results by repeating the results of the research.

\section{Limitations of the study}

By inclusion criteria, we should include patients with only a history of MA use. It has been extremely difficult to find a MA-consuming patient who has not used any other addictive substance in the past three months.

On the other hand, comorbid psychiatric disorders, along with dependence on substance abuse, is a common feature among patients with substance use disorders. A larger sample size was needed to understand the difference between the effects of comorbid psychiatric disorders, demographic conditions, and other items that were not significant in this study.

\section{CONCLUSION}

Modafinil is available in pills of $100 \mathrm{mg}$ and is tolerable to methamphetamine dependent outpatients during the withdrawal period. Given the promising results of the previous studies and the significant results of the recent study, it seems necessary to conduct a double-blind trial in the future with a large sample size and adequate dosage of modafinil and the appropriate treatment period. To the documented findings of this research, modafinil is safely indicated as an effective medication to reduce withdrawal symptoms and reduce craving.

\section{DECLARATIONS}

\section{Acknowledgement}

This research was supported by the Welfare Organization and the Ministry of Health of Iran. We gratefully acknowledge the clinical staff at Rasht's addiction treatment clinics that assisted with data collection.

\section{Conflict of interest}

Authors declare that no conflict of interest is associated with this work. There was no outside funding for this study. The authors have no disclosures or conflicts of interest.

\section{Contribution of authors}

We declare that this work was done by the authors named in this article and all liabilities pertaining to claims relating to the content of this article will be borne by the authors. The conceiving and designing of this study performed by Dr Mahnoosh Tavakkoli Fard, Dr Saeid Sadat Mansouri. Dr Nasim Vousooghi contributed to providing a critical revision of the article. Dr Alireza Jafari approving the final version of the manuscript, and Dr Saeid Sadat Mansouri wrote the manuscript. 


\section{Open Access}

This is an Open Access article that uses a funding model which does not charge readers or their institutions for access and distributed under the terms of the Creative Commons Attribution License (http://creativecommons.org/licenses/by/ 4.0) and the Budapest Open Access Initiative (http://www.budapestopenaccessinitiative.org/rea d), which permit unrestricted use, distribution, and reproduction in any medium, provided the original work is properly credited.

\section{REFERENCES}

1. Moeller SJ and Goldstein RZ. Impaired self-awareness in human addiction: deficient attribution of personal relevance Trends Cogn Sci 2014;18(12):635-641

2. Carroll KM and Rounsaville BJ. A vision of the next generation of behavioral therapies research in the addictions Addiction 2007;102(6):850-862

3. Uguen $M$, Perrin $D$, Belliard S, Ligneau $X$, Beardsley $P$, Lecomte $J$ and Schwartz J. Preclinical evaluation of the abuse potential of pitolisant, a histamine receptor inverse agonist antagonist compared with modafinil $\mathrm{Br} \mathrm{J}$ Pharmacol 2013;169(3):632-644

4. Organization $\mathrm{WH}$ and Sciences CfIOoM. International ethical guidelines for health-related research involving humans. Geneva: Council for International Organizations of Medical Sciences 2016

5. Dackis CA, Kampman KM, Lynch KG, Pettinati HM and O'brien CP. A double-blind, placebo-controlled trial of modafinil for cocaine dependence Neuropsychopharmacology 2005;30(1):205

6. Dunlop AJ, Brown AL, Oldmeadow C, Harris A, Gill A, Sadler C, Ribbons K, Attia J, Barker D and Ghijben $P$. Effectiveness and cost-effectiveness of unsupervised buprenorphine-naloxone for the treatment of heroin dependence in a randomized waitlist-controlled trial Drug Alcohol Depen 2017;174:181-191

7. Reichel CM and See RE. Modafinil effects on reinstatement of methamphetamine seeking in a rat model of relapse Psychopharmacology 2010;210(3):337-346

8. McGregor C, Srisurapanont M, Mitchell A, Wickes $W$ and White JM. Symptoms and sleep patterns during inpatient treatment of methamphetamine withdrawal: a comparison of mirtazapine and modafinil with treatment as usual J Subst Abus 2008;35(3):334-342

9. Kampman KM, Lynch KG, Pettinati HM, Spratt $K$, Wierzbicki MR, Dackis C and O'Brien CP. A double blind, placebo-controlled trial of modafinil for the treatment of cocaine dependence without co-morbid alcohol dependence Drug Alcohol Depen 2015;155:105110

10. Wisor JP. Modafinil as a catecholaminergic agent: empirical evidence and unanswered questions Front Neurol 2013; 4:139

11. Anderson AL, Reid MS, Li S-H, Holmes T, Shemanski L, Slee $A$, Smith EV, Kahn $R$, Chiang $N$ and Vocci $F$. Modafinil for the treatment of cocaine dependence Drug and alcohol dependence 2009;104(1-2):133-139

12. Heinzerling KG, Swanson A-N, Kim S, Cederblom L, Moe $A$, Ling $W$ and Shoptaw S. Randomized, double-blind, placebo-controlled trial of modafinil for the treatment of methamphetamine dependence Drug Alcohol Depen 2010;109(1-3):20-29

13. González B, Raineri M, Cadet JL, García-Rill E, Urbano FJ and Bisagno $V$. Modafinil improves methamphetamine-induced object recognition deficits and restores prefrontal cortex ERK signaling in mice Neuropharmacology 2014;87:188-197

14. Kalechstein AD, De La Garza I, Richard and Newton TF. Modafinil administration improves working memory in methamphetamine-dependent individuals who demonstrate baseline impairment The American journal on addictions 2010;19(4):340-344

15. Lee $N$, Pennay A, Hester R, McKetin R, Nielsen $S$ and Ferris J. A pilot randomised controlled trial of modafinil during acute methamphetamine withdrawal: feasibility, tolerability and clinical outcomes Drug and alcohol review 2013;32(1):88-95

16. Lee $N$, Pennay A, Hester R, McKetin R, Nielsen $S$ and Ferris J. A pilot randomised controlled trial of modafinil during acute methamphetamine withdrawal: feasibility, tolerability and clinical outcomes Drug Alcohol Rev 2013;32(1):88-95

17. McElhiney MC, Rabkin JG, Rabkin $R$ and Nunes EV. Provigil (modafinil) plus cognitive behavioral therapy for methamphetamine use in HIV gay men: a pilot study Am J Drug Alcohol Ab 2009;35(1):34-37

18. Rabkin JG, Gordon PH, McElhiney M, Rabkin R, Chew S and Mitsumoto $H$. Modafinil treatment of fatigue in patients with ALS: a placebo-controlled study Muscle \& Nerve 2009;39(3):297-303

19. Shearer J, Darke S, Rodgers C, Slade T, Van Beek I, Lewis J, Brady D, McKetin R, Mattick RP and Wodak A. A double-blind, placebo-controlled trial of modafinil for methamphetamine dependence Addiction 2009;104(2):224-233

20. Karila L, Weinstein A, Aubin HJ, Benyamina A, Reynaud $M$ and Batki SL. Pharmacological approaches to methamphetamine dependence: a focused review $\mathrm{Br}$. J. Clin. Pharmacol. 2010;69(6):578-592 